\title{
Novel targeted bladder drug-delivery systems: a review
}

This article was published in the following Dove Press journal:

Research and Reports in Urology

23 November 2015

Number of times this article has been viewed

\section{Martino Maria Zacchè Sushma Srikrishna Linda Cardozo}

Department of Urogynaecology, King's College Hospital, London, UK
Correspondence: Martino Maria Zacchè Department of Urogynaecology, King's College Hospital, Denmark Hill, London SE5 9RS, UK

Email m.zacche@gmail.com
Abstract: The objective of pharmaceutics is the development of drugs with increased efficacy and reduced side effects. Prolonged exposure of the diseased tissue to the drug is of crucial importance. Drug-delivery systems (DDSs) have been introduced to control rate, time, and place of release. Drugs can easily reach the bladder through a catheter, while systemically administered agents may undergo extensive metabolism. Continuous urine filling and subsequent washout hinder intravesical drug delivery (IDD). Moreover, the low permeability of the urothelium, also described as the bladder permeability barrier, poses a major challenge in the development of the IDD. DDSs increase bioavailability of drugs, therefore improving therapeutic effect and patient compliance. This review focuses on novel DDSs to treat bladder conditions such as overactive bladder, interstitial cystitis, bladder cancer, and recurrent urinary tract infections. The rationale and strategies for both systemic and local delivery methods are discussed, with emphasis on new formulations of well-known drugs (oxybutynin), nanocarriers, polymeric hydrogels, intravesical devices, encapsulated DDSs, and gene therapy. We give an overview of current and future prospects of DDSs for bladder disorders, including nanotechnology and gene therapy.

Keywords: drug targeting, drug-delivery system, bladder disorders

\section{Introduction}

Drug targeting is the ability of a drug to accumulate in a specific organ or tissue selectively and quantitatively, independently of the site and methods of its administration. This concept has been suggested by Paul Ehrlich a century ago. He spread the idea of the "magic bullet" for the synthesis of antibacterials. This was represented by the arsphenamine, the first truly effective drug against syphilis, and marketed under the name Salvarsan ("the arsenic that saves") at the beginning of the 1910s. Targeted treatment has obvious advantages. Pharmaceutical therapies are often dosed systemically, while their targets may be a specific tissue or a single compartment of the body. Therefore, the drug is distributed within the body and also has to cross biological barriers. Large quantities of drugs have to be administered to achieve a therapeutic concentration in a certain body compartment, also increasing the chances of negative side effects. It is understandable as to how research on drug targeting is under constant development and different delivery systems have been devised. The rationale is to improve efficacy by increasing the concentration at a target site while simultaneously minimizing toxicity by reducing off-target accumulation. ${ }^{2}$

An economic issue should also be addressed. In fact, the introduction of a new drug molecule in the market is expensive and time consuming, as it involves discovery, clinical testing, development, and regulatory approval. Improving safety/efficacy ratio of 
marketed drugs is more cost-effective. This can be obtained by controlling the rate, time, and place of release of drugs in the body through a drug-delivery system (DDS). Hence, DDS is an interface between the patient and the drug. It may be a formulation of the drug to administer it for a therapeutic purpose or a device used to deliver the drug. ${ }^{3}$

Selective pharmacologic targeting to individual compartments of the body is challenging. A therapeutic agent may be known, but its clinical application could be hindered by difficulties in achieving targeted delivery. Certain physiologic compartments such as the bladder, brain, and eye are viewed as "privileged" and may require specific techniques or devices to physically target drugs at the site of disease. ${ }^{4}$

Traditionally, many genitourinary conditions have been treated with medications administered orally. To increase efficacy and patient compliance, alternative delivery systems have been devised. The bladder is easily accessible using a catheter or cystoscope through the urethra by a simple procedure that can be performed by health care practitioners or even by patients themselves (clean intermittent selfcatheterization).

This review focuses on novel DDS for bladder disorders such as overactive bladder (OAB), interstitial cystitis (IC), bladder cancer (BC), and recurrent urinary tract infections (UTIs).

\section{Functional anatomy of urinary bladder}

The bladder is a hollow elastic organ that accommodates and stores urine, and facilitates voiding at appropriate intervals.
Its size varies during filling, and normal capacity is $\sim 500 \mathrm{~mL}$. Inner aspect of the bladder is lined by the urothelium, which is crucial in order to design effective intravesical drug delivery (IDD). It is a stratified epithelium, also known as transitional epithelium, with a layer of unique "umbrella cells" forming the luminal surface. ${ }^{5}$ These cells can flatten and increase surface area with stretching. ${ }^{6}$ Umbrella cells are covered by rigid plaques, composed of four major uroplakins, UPIa (27 kDa), UPIb (28 kDa), UPII (15 kDa), and UPIII $(47 \mathrm{kDa}) .^{7}$ The impermeability of the urothelium is further augmented by glycosaminoglycans (GAGs), which form a mucin hydrophilic layer adherent to the luminal side. ${ }^{8}$ These GAGs form a very tight barrier, capable of facing potentially noxious levels of urea, ammonia, and other toxic metabolites for prolonged periods of time. The function of this barrier is similar to the blood-brain barrier, with the only difference that the bladder is a storage organ, and needs to be more resistant to the passage of molecules. This is also known as the bladder permeability barrier (BPB) and represents an important obstacle in the success of IDD (Figure 1). ${ }^{9}$

\section{DDSs in bladder disease}

Novel DDSs have been devised to treat major bladder disorders. These are briefly summarized in the following paragraphs.

\section{Overactive bladder}

$\mathrm{OAB}$ is a symptom syndrome which is defined by the presence of urgency, with or without urgency incontinence, usually accompanied by frequency and nocturia in the absence

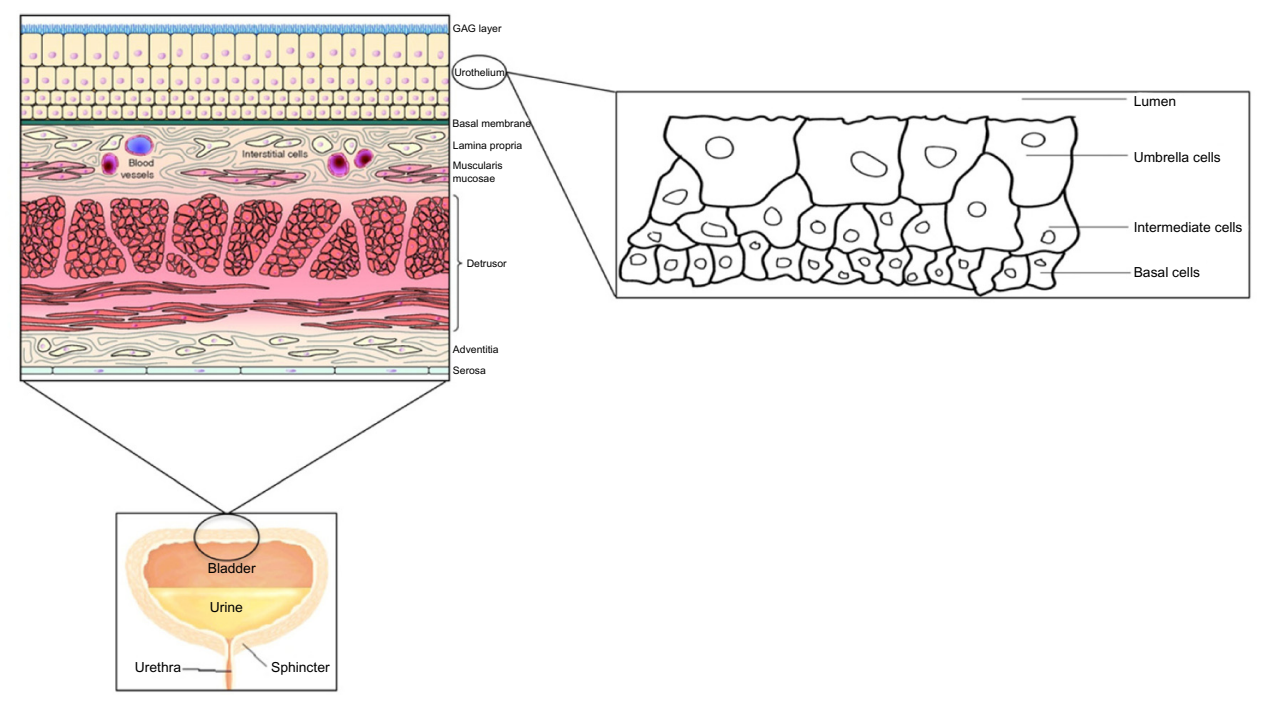

Figure I Structure of the urinary bladder wall and urothelium. Abbreviation: GAG, glycosaminoglycan. 
of infection or other obvious pathology. ${ }^{10}$ Antimuscarinics are the mainstay of current medical management, but the onset of side effect may lead the patient to discontinue the treatment. ${ }^{11}$ Research and development of novel therapeutic options is an active field in order to reduce the prevalence of refractory OAB. ${ }^{12,13}$

\section{Interstitial cystitis}

IC, or painful bladder syndrome, is a chronic condition seen mainly in women (10:1 female:male ratio). Main presenting symptoms are frequency, urgency, nocturia, and bladder pain, which often increases as the bladder fills and reduces after micturition. ${ }^{14}$ The pathophysiology is poorly understood and considered multifactorial. Currently, the most acceptable theory is injury or dysfunction of the GAG layer. ${ }^{15}$ Current treatment involves dietary manipulation, pain management, bladder retraining, and medication. There are several oral or intravesical options with variable efficacy, and to date, there is no consensus regarding which treatment is best. Potentially, IDD could better restore the integrity of the urothelial membrane. ${ }^{16}$

\section{Bladder cancer}

$\mathrm{BC}$ is caused by the uncontrolled growth of cells that line the bladder wall. It is the seventh most common cancer in the UK and the ninth worldwide. ${ }^{17,18}$ Main risk factors are increasing age, smoking, and exposure to urothelial carcinogens. ${ }^{19,20}$ Transitional cell carcinomas account for $>90 \%$ of tumors, while squamous cell carcinoma and adenocarcinoma represent $\sim 5 \%$ and $1 \%$, respectively. Symptoms may include hematuria, frequency, urgency, and dysuria. Seventy-five percent to $80 \%$ of $\mathrm{BCs}$ present as non-muscle-invasive disease, which can be managed with intravesical therapy. In these cases, either a chemotherapeutic drug or immunotherapy with Bacillus Calmette-Guérin may be given transurethrally. Novel DDSs could increase the stay of the drug in the bladder and potentially improve efficacy and therefore survival rate of the disease.

\section{Recurrent urinary tract infections}

UTI is one of the most common infectious diseases worldwide. Ten percent to $15 \%$ of all women will experience recurrent UTIs. ${ }^{21}$ These are defined as three or more episodes of UTI during a 12-month period or two infections in a 6-month period. ${ }^{22}$

Escherichia coli, the species most commonly involved in UTIs, can invade the urothelium and establish reservoirs in the cytoplasm of apical cells. ${ }^{23}$ Systemic antibiotics may not achieve effective doses in the bladder and also fail to penetrate the urothelium to eradicate intracellular reservoirs. Moreover, in the long-term, they can lead to antimicrobial resistances, side effects, and low patient compliance. Novel DDSs could allow higher concentrations of antibiotics in the bladder in order to pass through the urothelial cell membranes.

\section{Targeted bladder DDSs}

A DDS is a formulation or a device that enables the introduction of a therapeutic substance in the body and improves its efficacy and safety by controlling the rate, time, and place of release of drugs in the body. It involves the administration of the therapeutic product, the release of the active ingredients by the product, and the subsequent transport of the active ingredients across the biological membranes to the site of action. A significant amount of work in drug delivery targeted to the bladder has focused on the disorders mentioned above. Drugs may be either administered directly to the bladder, or given systemically and targeted to the bladder. Most of the drugs available for treatment of bladder diseases are administered orally. The reasons for this preference are the ease of administration and widespread acceptance by patients. The challenge consists in delivering an effective dose in the bladder minimizing undesired biodistribution which could increase side effects. Different strategies have been developed to find drugs with better efficacy and tolerability, leading to novel therapeutic options. The bladder can be easily accessed using a catheter or cystoscope through the urethra. Hence, it is an attractive local drug-delivery target.

\section{Systemic delivery methods}

A classification of various methods of systemic drug delivery by anatomical routes is shown in Table 1 .

Table I A classification of various anatomical routes for systemic drug delivery
Gastrointestinal system
Oral
Rectal
Parenteral
Subcutaneous injection
Intramuscular injection
Intravenous injection
Intra-arterial injection
Transmucosal
Transnasal
Pulmonary
Transdermal
Intra-osseous infusion 
New formulations of well-known drugs are in constant development in order to minimize side effects and improve tolerability. For example, oxybutynin is the most widely prescribed antimuscarinic for $\mathrm{OAB} .{ }^{24}$ Oxybutynin is a tertiary amine, and when administered orally, undergoes extensive upper gastrointestinal and first-pass hepatic metabolism via the cytochrome P450 system. Its primary metabolite is $N$-desethyloxybutynin ( $N$-DEO) and is the major contributor to the occurrence of dry mouth. ${ }^{25}$ Extended-release formulations when compared to immediate-release cause less reduction in saliva production, a typical side effect of antimuscarinics. ${ }^{26}$

\section{Transdermal route}

As an alternative to oral route, transdermal drug delivery of oxybutynin has been introduced, and the US Food and Drug Administration (FDA) approved it in 2003. Not surprisingly, reducing first-pass metabolism, the transdermal route has shown better tolerability. ${ }^{27,28}$ Transdermal patches were the first to be marketed, followed by transdermal gel, with the aim of reducing application site reactions as erythema and pruritus. ${ }^{29}$ Penetration enhancers have also been used to facilitate transdermal drug transport. Recently, Goldfischer et al published the results of a Phase III trial that looked at the efficacy and safety of oxybutynin transdermal gel 3\% (OTG3\%) formulated with propylene glycol as permeation enhancer. OTG3\% $84 \mathrm{mg}$ and OTG3\% $56 \mathrm{mg}$ were compared with placebo gel in adults with urgency and/or mixed urinary incontinence (UI). In December 2011, the FDA approved the availability of OTG3\% (84 mg/d) in a titratable pump dispenser, while the lower dose $(56 \mathrm{mg} / \mathrm{d})$ was not approved. Six hundred and twenty-six patients were randomized 1:1:1 in a double-blind, placebo-controlled, multicenter study. The primary efficacy end point was change from baseline to week 12 in weekly UI episodes recorded in 3-day bladder diaries. Secondary end points were change from baseline to week 12 in daily urinary frequency and change from baseline to week 12 in average urinary void volume. OTG3\% $84 \mathrm{mg} / \mathrm{d}$ achieved significantly greater improvement vs placebo in weekly UI episodes (mean change from baseline: -20.4 vs $-18.1, P<0.05)$, daily urinary frequency $(-2.6$ vs $-1.9, P=0.001)$, and urinary void volume (32.7 vs $9.8, P<0.0001)$. Dry mouth and application site erythema were more common with OTG3\% $84 \mathrm{mg} / \mathrm{d}$ than with placebo $(12.1 \%$ vs $5.0 \%, P=0.028$, and $3.7 \%$ vs $1.0 \%, P=\mathrm{NS}$, respectively). Changes from baseline to week 12 of OTG3\% $56 \mathrm{mg} / \mathrm{d}$ vs placebo were not significant for primary and secondary outcomes. ${ }^{30}$

\section{Vaginal route}

The vaginal route represents an alternative for women who are unable to tolerate adverse effects of oral antimuscarinics or application site reactions associated with topical/transdermal products. Teva Pharmaceutical Industries Ltd introduced a flexible transvaginal ring that is designed to continuously release oxybutynin (DR-3001). In view of the promising results of a Phase II trial, ${ }^{31}$ DR-3001 underwent a Phase III evaluation. One thousand one hundred and two women with pure-predominantly urge UI were randomized to DR-3001 $4 \mathrm{mg} / \mathrm{d}$, DR-3001 $6 \mathrm{mg} / \mathrm{d}$, or placebo vaginal ring, inserted vaginally and replaced every 4 weeks. In this multicenter, 12-week trial, the primary end point was the change in total weekly number of UI episodes. Secondary outcomes were change in average daily urinary frequency, void volume, and proportion of subjects with no UI episodes. These were recorded in a final 3-day diary. DR-3001 $4 \mathrm{mg} / \mathrm{d}$ and $6 \mathrm{mg} / \mathrm{d}$ showed significantly greater improvement vs placebo in weekly UI episodes (mean change from baseline: -20.5 and -21.4 vs $-18.1, P=0.0013$ and $P<0.0001$, respectively) and daily urinary frequency (mean change from baseline: -2.0 and -3.2 vs $-2.0, P=0.0005$ and $P<0.0001$, respectively). No significant differences were noted between DR-3001 $4 \mathrm{mg} / \mathrm{d}$ and $6 \mathrm{mg} / \mathrm{d}$ vs placebo in terms of urinary void volume (mean change from baseline: 25.4 and 23.6 vs 18.8, P=NS). DR-3001 $4 \mathrm{mg} / \mathrm{d}$ and $6 \mathrm{mg} / \mathrm{d}$ also had a greater proportion of women with no UI episodes recorded in the final 3-day diary (36\% and 35\% vs $28 \%, P=0.029$ and $P=0.074$, respectively). Except for a higher incidence of dry mouth, DR-3001 was well tolerated and had a safety profile similar to that of the placebo ring. In fact, dry mouth occurred in $6 \%$ of cases with DR-3001 $4 \mathrm{mg} / \mathrm{d}, 8 \%$ with DR-3001 $6 \mathrm{mg} / \mathrm{d}$, and 4\% with placebo; constipation occurred in $1 \%, 1 \%$, and $2 \%$ of cases with DR-3001 $4 \mathrm{mg} / \mathrm{d}$, DR-3001 $6 \mathrm{mg} / \mathrm{d}$, and placebo, respectively; culture-positive UTIs occurred in $8 \%, 10 \%$, and $8 \%$ of cases with DR-3001 $4 \mathrm{mg} / \mathrm{d}$, DR-3001 $6 \mathrm{mg} / \mathrm{d}$, and placebo, respectively. ${ }^{32}$

\section{Local delivery methods}

Bladder anatomy allows relatively easy and direct delivery of drugs through a catheter, allowing the drug to reach the target site with minimal side effects while avoiding firstpass metabolism. Intravesical administration of oxybutynin compared to oral showed higher bioavailability, with reduced concentration of its active metabolite $\mathrm{N}$-DEO. ${ }^{33}$

Dysfunction of the GAG layer is the most currently accepted theory for IC. Hence, the use of IDD might be rational because oral therapy requires large doses and only a small portion is actually absorbed and reaches the bladder. ${ }^{34,35}$ 
The intravesical administration of hyaluronic acid (HA) has been shown to be effective in the IC, as it replaces GAG layer. ${ }^{36}$ Moreover, it inhibits leukocyte aggregation and migration, and adherence of immune complexes to polymorphonuclear cells. ${ }^{37}$ Recent studies demonstrated how HA significantly suppresses the secretion of pro-inflammatory cytokines interleukin (IL)-6 and IL-8 and also increases the levels of sulfated GAGs. ${ }^{38}$

However, DDSs are also associated with some difficulties. Firstly, drug dilution occurs as the urine is continuously produced and due to subsequent washout during urine voiding. Secondly, the urothelium forms a low permeability barrier, allowing poor penetration of drugs into the bladder wall. Thirdly, repetitive catheterization may reduce patient compliance. To overcome this matter, different strategies have been employed. Drugs may be injected into the bladder wall. For example, in cases of OAB refractory to antimuscarinics, intradetrusorial injection of onabotulinumtoxin A (Botox ${ }^{\circledR}$; Allergan Inc., Irvine, CA, USA) is currently routine practice in most of the units dealing with lower urinary tract dysfunctions. ${ }^{39}$ Other approaches to increase drug permeability are physical methods and chemical permeation enhancers (Table 2).

Novel targeted DDSs have recently been developed to prolong the effect of drugs in the bladder. This has been possible with the introduction of nanotechnology in the field of drug delivery. This science refers to structures that have one or more dimensions in the nanometer scale. ${ }^{41}$ Substances are loaded within nanoparticles. ${ }^{42}$ This holds a high potential of improving the therapeutic value of different drugs by increasing bioavailability, solubility, and retention time. ${ }^{43}$

\section{Nanocarriers}

Nanocarriers can deliver drugs to site-specific targets. They can be formulated from materials such as lipids, polymers, proteins, and metals. ${ }^{44}$

Liposomes are phospholipid concentric bilayer nanospheres mimicking human cell walls. They allow the delivery of both lipophilic drugs such as capsaicin and hydrophilic

Table 2 Strategies for improving drug permeability

Physical approaches
Electromotive drug administration
Hyperthermia (intravesical mitomycin C instillation) ${ }^{40}$
Chemical approaches
Dimethyl sulfoxide
Protamine sulfate
Biomolecules (chitosan, polycarbophil)

drugs such as botulinum toxin. Clathrin-mediated endocytosis is their route of entry into the urothelium..$^{45}$

Intravesical administration of liposomes alone can enhance resistance to irritant penetration as shown in a rat model with hyperactive bladder. ${ }^{46}$ Further studies showed that intravesical liposomes are safe and have similar efficacy to one of the most effective FDA-approved pharmacological treatments for IC as oral pentosan polysulfate (PP) $\left(\right.$ Elmiron $\left.^{\circledR}\right)$. Empty liposomes have been shown to be effective as potential treatment of IC. ${ }^{47}$ This is thought to be related to their ability to form a protective lipid film on the urothelial surface. In a pilot study, intravesical instillation of PP encapsulated in liposomes was effective in refractory IC patients. ${ }^{48}$ However, studies with bigger number and placebo groups are warranted.

Liposomal vesicles enhance the therapeutic index of chemotherapeutic agents and represent a promising approach for cancer treatment. ${ }^{49}$ Currently, a Phase II trial is evaluating temperature-sensitive liposomes plus local hyperthermia in patients with recurrent chest wall breast cancer. ${ }^{50}$ Encouraging results could lead to explore this approach in different clinical setting, including BC.

Other nanocarriers that can be used for IDD are solid lipid nanoparticles, protein nanoparticles with targeted ligands grafted on the surface, branched polymeric dendrimers, mucoadhesive biopolymers (chitosan) or synthetic polymers, magnetic particles, gold nanoshells, and in situ gelling systems. ${ }^{51}$

\section{Polymeric hydrogels}

A novel approach is the use of hydrogels as depot formulations on the bladder walls. This enables longer exposure of the urinary tract tissue to existing drugs, as compared to standard intravesical instillation, as they remain attached to the bladder wall even after urine voiding. ${ }^{52}$ Between the different types that have been developed, thermosensitive hydrogels have gained increasing attention..$^{53}$ These are aqueous solutions of poly (ethylene glycol-b-[DL-lactic acid-co-glycolic acid]-b-ethyleneglycol) triblock copolymers that form a freeflowing solution at room temperature and become a viscous gel at body temperature of $37^{\circ} \mathrm{C} .{ }^{54}$

There are several potential polymeric gel systems that have been explored. $\mathrm{TCGel}^{\circledR}$ is a novel hydrogel with reverse thermal gelation properties produced by TheraCoat Ltd (Raanana, Israel). This means that it is fluid when cold and a gel at body temperature. Following instillation, the gel solidifies and acts as sustained drug release depot in situ. When the gel is in contact with urine, it dissolves and gradually 
releases the drug over a period of 6-8 hours. TCGel ${ }^{\circledR}$ is slowly excreted from the bladder during urination. It is $100 \%$ biocompatible and harmless to the body. TheraCoat Ltd has commenced several efficacy clinical trials.

A Phase I/II clinical trial was completed in Israel exploring the feasibility, tolerability, and safety of 200 units of onabotulinumtoxin A (Botox ${ }^{\circledR}$ ) premixed with chilled $40 \mathrm{~mL}$ of TCGel ${ }^{\circledR}$. Fifteen patients with IC were enrolled in the trial and received a single instillation. Follow-up was at weeks 2 , 6 , and 12 post-instillation. The efficacy outcome measures included IC Symptom Index (ICSI) and IC Problem Index (ICPI). A reduction in the ICSI and ICPI was observed since week 2 and was also apparent at week 12. Likewise, a reduction from baseline was observed in the daytime urinary frequency and urge episodes, while the nocturnal frequency remained unchanged. No serious drug-related adverse events were reported. ${ }^{55}$

TheraCoat Ltd also completed the Intravesical Botulinum Toxin Treatment for OAB (INTIMO) study, a double-blind placebo-controlled study aiming to evaluate the efficacy of bladder instillation with botulinum toxin and $\mathrm{TCGel}^{\circledR}$ in patients with $\mathrm{OAB}$, but the results are not as yet available. ${ }^{56}$

Other ongoing clinical trials are currently looking at the intravesical instillation of mitomycin $\mathrm{C}$ (MMC) mixed with TCGel $^{\circledR}$ in patients with non-muscle-invasive BC (Optimized Instillation of Mitomycin for bladder cancer treatment [OPTIMA] study). ${ }^{57}$ Preclinical results demonstrated the following:

- levels of MMC in the bladder 2 hours and 6 hours following instillation were over tenfold higher as compared to drug alone (same total dosage);

- levels of MMC in the urine were higher for a longer period of time compared to drug alone;

- levels of MMC in the plasma were low, confirming safety of the treatment.

For intravesical chemotherapy, hydrophobic anticancer drugs offer a distinct advantage of greater permeability through the urothelium as compared to hydrophilic drugs. This allows longer retention of therapeutic doses within the bladder tissue even after the instilled solution is washed out after voiding. Loading hydrophobic drugs into carriers for sustained IDD is viewed as one of the most promising strategies to ensure continuous presence of drug in the bladder with fewer or no intermittent catheterization. Mucoadhesive nanogels have been recently evaluated in porcine urinary bladder and could be a promising candidate for intravesical delivery of hydrophobic drugs in $\mathrm{BC}$ therapy. ${ }^{58}$

\section{Intravesical devices}

Intravesical instillation therapy has a number of potential benefits including high drug concentration at the bladder and reduced systemic exposure which minimizes systemic toxicity and side effects compared to oral medications. However, repeated instillations are often required to extend the drug exposure, and this lowers the compliance. Indwelling intravesical devices are physical devices that can be inserted and remain in the bladder. The drug is then released from the device in a controlled and extended manner. The device can be either biodegradable or nondegradable. The former eliminates the device removal step but can provoke debris that might lead to voiding difficulties.

A reservoir-based device constructed of a completely biodegradable elastomer supplying ciprofloxacin- $\mathrm{HCl}$ has been evaluated in vitro and showed functionality and stability. ${ }^{59}$ Biodegradable devices have also been studied for IDD of antimuscarinics as this could represent an alternative to oral treatment for OAB. In vitro studies in a porcine bladder model showed effectiveness of trospium chloride $(\mathrm{TrCl})$ when loaded onto degradable poly(lactide-co-glycolide)-based polymer carriers. ${ }^{60}$ Lipid-based intravesical DDSs were loaded with $\mathrm{TrCl}$ and showed a drug release ranging from several days up to weeks. ${ }^{61}$

Trials with nondegradable indwelling devices first evaluated an intravesical pump called UROS, developed by Situs Corporation Ltd (Houston TX, USA). The device was tested in Phase I/II trials. ${ }^{62}$ However, no more recent trials have been published.

A nonbiodegradable device, Lidocaine Releasing Intravesical System (LiRIS ${ }^{\circledR}$; TARIS Biomedical, Lexington, MA, USA), has been deemed for intravesical delivery of lidocaine. It has a double design: one tube contains solid lidocaine hydrochloride minitablets, enabling a higher dose of drug than if a solution was used, and the other lumen contains nickel titanium wire, which adopts a "pretzel" conformation once inside the bladder, preventing it from being accidentally voided. It is retained in the bladder with minimal discomfort, allowing release of lidocaine for a sustained period. A silicone container absorbs urine to dissolve the lidocaine contents, whereas the osmotic pressure created forces the solution out of the container through a small orifice in a controlled release over 14 days. Promising results were achieved when the device was tried in a rabbit model. In fact, lidocaine concentration in the bladder tissue was $>0.1 \mu \mathrm{g} / \mathrm{g}$ during the 3 -day period of device release, while a single instillation yielded immeasurable amounts within 24 hours. ${ }^{63}$ 
TARIS Biomedical carried out a Phase I safety assessment study in healthy volunteers. An empty device was inserted into the bladder of seven women, while three women underwent cystoscopy without placement of the device. Tolerability was assessed using visual analog scales for bladder sensation and voiding behavior, which revealed that changes from baseline were similar between groups. A Phase Ib cohort study in 16 women with moderate-tosevere IC followed. Subjects received either LiRIS ${ }^{\circledR} 200 \mathrm{mg}$ or LiRIS ${ }^{\circledR} 650 \mathrm{mg}$ for 2 weeks. There was a significant improvement in bladder pain, urinary urgency, and voiding frequency. Cystoscopic findings also improved on day 14 (day of removal) compared with day 1. Most strikingly, of the six patients who were noted to have Hunner's lesion on cystoscopic insertion, five demonstrated complete resolution 14 days later. Global response assessment showed an overall responder rate of $64 \%$ at day 14 and a sustained overall responder rate of $64 \% 2$ weeks later. Extended follow-up suggests that the reduction in pain was maintained for several months after the device was removed. Expanded Phase II/ III trials are now underway. Potentially, other therapeutic agents could be delivered by the device for the treatment of other bladder disorders. ${ }^{64}$

Allergan Inc. is sponsoring two Phase II clinical trials that aim to establish the safety and efficacy of LiRIS ${ }^{\circledR}$ in IC patients with ${ }^{65}$ and without ${ }^{66}$ Hunner's lesions.

\section{Encapsulated DDS}

There are pilot data showing the feasibility of loading an antibiotic into a non-cell-toxic microcapsule that can both penetrate bladder cells and provide slow release of the drug that retains its bactericidal properties. ${ }^{67}$ This system has been developed for the delivery of high doses of antibiotic to treat recalcitrant UTIs. The capsules have been prepared with coaxial electrohydrodynamic forming technology, which has shown considerable promise for the production of fibers and particles at the micro- and nanoscale. ${ }^{68}$ Polymethylsilsesquioxane has showed good biocompatibility and physiological inertness and has been inserted in the capsules together with gentamicin. ${ }^{69}$ In an in vitro assay, the capsules were able to kill Enterococcus faecalis, the most common uropathogen. Moreover, the capsules were incubated with bladder epithelial cells collected from the urine of patients with UTIs. Successful delivery in bladder cells was demonstrated, but the mechanism is still unknown. High localized doses of antibiotic could be reached by introducing the capsules directly into the bladder via a catheter.

\section{Gene therapy}

Gene therapy is a promising therapy for controlling $\mathrm{BC}$ as it is highly selective for tumor cells with mutated genes. Moreover, it carries the potential of restoring normal cell growth by correcting genetic defects. Gene delivery systems include both viral (adenovirus) and nonviral vectors (lipoplexes).

In viral vectors, the genome contains the therapeutic gene that is carried into the mutated cells. Viral receptor expression and urothelium represent barriers that research is trying to overcome. Viral vectors are associated with permeation enhancers to improve the delivery of intravesical gene therapy. Modulation of coxsackie-adenovirus receptor expression $^{70}$ and coadministration of polyamide Syn 3 are active research fields. ${ }^{71}$ Although the use of Syn 3 appears to be nontoxic to the bladder tissue, other transductionenhancing agents may produce adverse effects on the urothelium..$^{72,73}$

Nonviral vectors consist of genes incorporated into liposomes, such as IL-12 gene, to induce immune response. The interest toward these vectors has increased due to the potential of toxicity from systemic absorption of viral vectors from the bladder. ${ }^{74}$

Small "interfering" RNAs delivered into the bladder using liposomes have been effective in vivo and may represent a novel therapeutic tool. ${ }^{75}$ Gene therapy has also been developed to treat $\mathrm{OAB}$ and IC in rat models and may represent a future treatment modality. ${ }^{76}$ From epidemiologic studies, there is increasing evidence that bladder disorders as UI and $\mathrm{OAB}$ are heritable conditions. Underlying genetic variants have been identified and could potentially represent future drug targets. ${ }^{77}$

\section{Conclusion}

The goal of targeted delivery drugs is to transport proper amounts of drugs to the desirable sites while minimizing unwanted side effects on other tissues. Several strategies have been developed within the last decade. Various drug formulations, routes of drug administration, as well as devices for drug delivery and targets have been described. The bladder is an ideal target for local drug delivery as it is easily accessed through the urethra. IDD is however hindered by continuous filling of the bladder with urine, the low permeability of the BPB, and the washout during voiding. Hence, serial drug instillations are often required.

In the last decade, novel DDSs have been devised with the aim to improve the therapeutic effect and reduce costs linked to the discovery of new drugs. Nanotechnology has 
revolutionized the field of drug delivery and represents a great potential for the future. This will require interdisciplinary teams including clinicians, biologists, engineers, and pharmaceutical scientists. Gene therapy also has advantages as it is highly specific for tumor cells. Overall, further clinical trials are required to move this therapeutic option into the clinical setting.

\section{Disclosure}

MM Zacche reports no conflicts of interest in this work. S Srikrishna received funding from Astellas. L Cardozo, during the last year, received funding for research, lecturing and/or advice/consultancies from Allergan, Astellas, and Pfizer. The authors report no other conflicts of interest in this work.

\section{References}

1. Torchilin VP. Drug targeting. Eur J Pharm Sci. 2000;11(Suppl 2): S81-S91.

2. Nirmal J, Chuang YC, Tyagi P, Chancellor MB. Intravesical therapy for lower urinary tract symptoms. Urol Sci. 2012;23:70-77.

3. Butte K, Momin M, Kurhade S, Kar S. Intravesical drug delivery system for bladder: an overview. Int J Pharm Chem Biol Sci. 2013; 3(3):680-691.

4. Cima MJ, Lee H, Daniel K, et al. Single compartment drug delivery. J Control Release. 2014;28(190):157-171.

5. Lewis SA. Everything you wanted to know about the bladder epithelium but were afraid to ask. Am J Physiol Renal Physiol. 2000; 278(6):867-874.

6. Lewis SA. The mammalian urinary bladder: it's more than accomodating. News Physiol Sci. 1986;1:61-65.

7. Boen M, Pahner I, Ahnert-Hilger G, Jons T. The mantainance of the permeability barrier of bladder facet cells requires a continuous fusion of discoid vesicles with the apical plasma membrane. Eur J Cell Biol. 2003;82:343-350.

8. Hurst RE. Structure, function, and pathology of proteoglycans and glycosaminoglycans in the urinary tract. World J Urol. 1994;12(1):3-10.

9. Tyagi P, Wu PC, Chancellor M, Yoshimura N, Huang L. Recent advances in intravesical drug/gene delivery. Mol Pharm. 2006;3(4): 369-379.

10. Abrams P, Cardozo L, Fall M, et al; Standardisation Sub-committee of the International Continence Society. The standardisation of terminology of lower urinary tract function: report from the standardisation sub-committee of the international continence society. Neurourol Urodyn. 2002;21:167-178.

11. Benner JS, Nichol MB, Rovner ES, et al. Patient-reported reasons for discontinuing overactive bladder medication. BJU Int. 2010;105: 1276-1282.

12. Schwantes U, Grosse J, Wiedemann A. Refractory overactive bladder: a common problem? Int Urogynecol J. 2015;26(10):1407-1414.

13. Zacche MM, Giarenis I, Cardozo L. Phase II drugs that target cholinergic receptors for the treatment of overactive bladder. Expert Opin Investig Drugs. 2014;23(10):1365-1374.

14. McLennan MT. Interstitial cystitis: epidemiology, pathophysiology, and clinical presentation. Obstet Gynecol Clin North Am. 2014; 41(3):385-395.

15. Parsons CL. The role of the urinary epithelium in the pathogenesis of interstitial cystitis/prostatis/urethritis. Urology. 2007;69(4 Suppl):9-16.

16. Tseng LH. Advances in the methods for discovering novel painful bladder syndrome therapies. Expert Opin Drug Discov. 2014;9(4):423-432.

17. NICE guideline. Bladder Cancer: Diagnosis and Management. NICE guideline; 2015. Available from: https://www.nice.org.uk/guidance/ng2. Accessed June 6, 2015.
18. Colombel M, Soloway M, Akaza H, et al. Epidemiology, staging, grading, and risk stratification of bladder cancer. Eur Urol Suppl. 2008;7: 618-626.

19. Parkin DM. Tobacco-attributable cancer burden in the UK in 2010. Br J Cancer. 2011;105(Suppl 2):S6-S13.

20. Parkin DM. Cancers attributable to occupational exposures in the UK in 2010. Br J Cancer. 2011;105:S70-S72.

21. Hooton TM. Recurrent urinary tract infection in women. Int J Antimicrob Agents. 2001;17(4):259-268.

22. Albert X, Huertas I, Pereiró II, Sanfélix J, Gosalbes V, Perrota C. Antibiotics for preventing recurrent urinary tract infection in nonpregnant women. Cochrane Database Syst Rev. 2004;3:CD001209.

23. Hunstad DA, Justice SS. Intracellular lifestyles and immune evasion strategies of uropathogenic Escherichia coli. Annu Rev Microbiol. 2010;64:203-221.

24. Jirschele K, Sand PK. Oxybutynin: past, present, and future. Int Urogynecol J. 2013;24(4):585-604.

25. Hughes KM, Lang JC, Lazare R, et al. Measurement of oxybutynin and its $\mathrm{N}$-desethyl metabolite in plasma, and its application to pharmacokinetic studies in young, elderly and frail elderly volunteers. Xenobiotica. 1992;22:859-869.

26. Sathyan G, Chancellor MB, Gupta SK. Effect of OROS $^{\circledR}$ controlledrelease delivery on the pharmacokinetics and pharmacodynamics of oxybutynin chloride. Br J Clin Pharmacol. 2001;52:409-417.

27. Davila GW, Daugherty CA, Sanders SW. A short term, multicenter, randomized double-blind dose titration study of the efficacy and anticholinergic effects of transdermal compared to immediate release oral oxybutynin treatment of patients with urge urinary incontinence. J Urol. 2001;166:140-145.

28. Staskin DR, Robinson D. Oxybutynin chloride topical gel: a new formulation of an established antimuscarinic therapy for overactive bladder. Expert Opin Pharmacother. 2009;10:3103-3111.

29. Pizzi LT, Talati A, Gemmen E, Dahl NV, Bunz TJ, Sand PK. Impact of transdermal oxybutynin on work productivity in patients with overactive bladder: results from the MATRIX study. Pharmacoeconomics. 2009;27:329-339.

30. Goldfischer ER, Sand PK, Thomas H, Peters-Gee J. Efficacy and safety of oxybutynin topical gel 3\% in patients with urgency and/or mixed urinary incontinence: a randomized, double-blind, placebo-controlled study. Neurourol Urodyn. 2015;34(1):37-43.

31. Gittelman M, Weiss H, Seidman L. A phase 2, randomized, doubleblind, efficacy and safety study of oxybutynin vaginal ring for alleviation of overactive bladder symptoms in women. $J$ Urol. 2014; 191(4):1014-1021.

32. Swierzewski M, Seidman L, Dasen S, Weiss H. Phase 3 Efficacy and safety of once-monthly oxybutynin vaginal ring delivering $4 \mathrm{mg} /$ day or $6 \mathrm{mg} /$ day vs placebo ring in women with urge incontinence, frequency, and urgency symptoms of overactive bladder [abstract 565]. 108th Annual Meeting of the American Urological Association; May 4-8. San Diego, CA: 2013.

33. Krause P, Fuhr U, Schnitker J, Albrecht U, Stein R, Rubenwolf P. Pharmacokinetics of intravesical versus oral oxybutynin in healthy adults: results of an open label, randomized, prospective clinical study. J Urol. 2013;190(5):1791-1797.

34. Colaco MA, Evans RJ. Current recommendations for bladder instillation therapy in the treatment of interstitial cystitis/bladder pain syndrome. Curr Urol Rep. 2013;14:442-447.

35. Neuhaus J, Schwalenberg T. Intravesical treatment of bladder pain syndrome/interstitial cystitis. Nat Rev Urol. 2012;9:707-720.

36. Lai MC, Kuo YC, Kuo HC. Intravesical hyaluronic acid for interstitial cystitis/painful bladder syndrome: a comparative randomized assessment of different regimens. Int J Urol. 2013;20:203-207.

37. Daha LK, Riedl CR, Lazar D, Hohlbrugger G, Pfluger H. Do cystometric findings predict the results of intravesical hyaluronic acid in women with interstitial cystitis? Eur Urol. 2005;47:393-397.

38. Rooney P, Srivastava A, Watson L, Quinlan LR, Pandit A. Hyaluronic acid decreases IL-6 and IL-8 secretion and permeability in an inflammatory model of interstitial cystitis. Acta Biomater. 2015;19:66-75. 
39. Kuo YC, Kuo HC. Botulinum toxin injection for lower urinary tract dysfunction. Int J Urol. 2013;20(1):40-55.

40. Colombo R, Salonia A, Leib Z, Pavone-Macaluso M, Engelstein D. Long-term outcomes of a randomized controlled trial comparing thermochemotherapy with mitomycin-C alone as adjuvant treatment for nonmuscle-invasive bladder cancer (NMIBC). BJU Int. 2011;107:912-918.

41. Park N. Nanotechnology: what it can do for drug delivery. J Control Release. 2007;120:1-3.

42. Harrington DA, Sharma AK, Erickson BA, Cheng EY. Bladder tissue engineering through nanotechnology. World J Urol. 2008;26(4): 315-322.

43. Kumari A, Yadav SK, Yadav SC. Biodegradable polymeric nanoparticles based drug delivery systems. Colloids Surf B Biointerfaces. 2010;75(1):1-18.

44. Singh R, Lillard JW Jr. Nanoparticle-based targeted delivery. Exp Mol Pathol. 2009;86(3):215-223.

45. Rajaganapathy BR, Chancellor MB, Nirmal J, Dang L, Tyagi P. Bladder uptake of liposomes after intravesical administration occurs by endocytosis. PLoS One. 2015;10(3):e0122766.

46. Fraser MO, Chuang YC, Tyagi P, et al. Intravesical liposome administration-a novel treatment for hyperactive bladder in the rat. Urology. 2003;61(3):656-663.

47. Chuang YC, Lee WC, Lee WC, Chiang PH. Intravesical liposome versus oral pentosan polysulfate for interstitial cystitis/painful bladder syndrome. J Urol. 2009;182(4):1393-1400.

48. Lander EB, See JR. Intravesical instillation of pentosan polysulfate encapsulated in a liposome nanocarrier for interstitial cystitis. Am J Clin Exp Urol. 2014;2(2):145-148.

49. Haley B, Frenkel E. Nanoparticles for drug delivery in cancer treatment. Urol Oncol. 2008;26(1):57-64.

50. ClinicalTrials.gov. Available from: http://www.clinicaltrials.gov/show/ NCT00826085. Last Accessed July 26, 2015.

51. GuhaSarkar S, Banerjee R. Intravesical drug delivery: challenges, current status, opportunities and novel strategies. $J$ Control Release. 2010;148(2):147-159.

52. Gong C, Qi T, Wei X, et al. Thermosensitive polymeric hydrogels as drug delivery systems. Curr Med Chem. 2013;20(1):79-94.

53. Tyagi P, Li Z, Chancellor M, De Groat WC, Yoshimura N, Huang L. Sustained intravesical drug delivery using thermosensitive hydrogel. Pharm Res. 2004;21:832-837.

54. Jeong B, Bae YH, Kim SW. In situ gelation of PEG-PLGA-PEG triblock copolymer aqueous solutions and degradation thereof. J Biomed Mater Res. 2000;50(2):171-177.

55. Stav K, Vinshtok Y, Jeshurun M, Ivgy-May N, Gerassi T, Zisman A. Safety and feasibility of intravesical instillation of botulinum toxin in hydrogel-based slow release delivery system in PBS/IC patients: a pilot study [abstract 163]. 44th Annual Meeting of the International Continence Society; October 20-24. Rio de Janeiro, Brazil: 2014.

56. Available from: http://theracoat.com/clinical-trials/. Last Accessed Jul 26, 2015.

57. ClinicalTrials.gov. Available from: http://www.clinicaltrials.gov/show/ NCT01803295. Last Accessed July 26, 2015.

58. Lu S, Neoh KG, Kang ET, Mahendran R, Chiong E. Mucoadhesive polyacrylamide nanogel as a potential hydrophobic drug carrier for intravesical bladder cancer therapy. Eur J Pharm Sci. 2015;72:57-69.

59. Tobias IS, Lee H, Engelmayr GC, Macaya D, Bettinger CJ, Cima MJ. Zero-order controlled release of ciprofloxacin- $\mathrm{HCl}$ from a reservoirbased, bioresorbable and elastomeric device. J Control Release. 2010 145(3):356-362.
60. Von Walter M, Michaelis I, Jakse G, Grosse JO. Trospium chloride released from intravesically applied PLGA-based carriers decreases bladder contractility in an insolated whole pig bladder model. Eur Urol Suppl. 2009;8:178.

61. Haupt M, Thommes M, Heidenreich A, Breitkreutz J. Lipid-based intravesical drug delivery systems with controlled release of trospium chloride for the urinary bladder. $J$ Control Release. 2013;170:161-166.

62. Fraser MO, Lavelle JP, Sacks MS, Chancellor MB. The future of bladder control-intravesical drug delivery, a pinch of pepper, and gene therapy. Rev Urol. 2002;4:1-11.

63. Lee H, Cima MJ. An intravesical device for the sustained delivery of lidocaine to the bladder. J Control Release. 2011;149:133-139.

64. Nickel JC, Jain P, Shore N, et al. Continuous intravesical lidocaine treatment for interstitial cystitis/bladder pain syndrome: safety and efficacy of a new drug delivery device. Sci Transl Med. 2012;4(143):143ra100.

65. ClinicalTrials.gov. Available from: http://www.clinicaltrials.gov/show/ NCT02395042. Last Accessed July 26, 2015.

66. ClinicalTrials.gov. Available from: http://www.clinicaltrials.gov/show/ NCT02411110. Last Accessed July 26, 2015.

67. Labbaf S, Horsley H, Chang MW, et al. An encapsulated drug delivery system for recalcitrant urinary tract infection. $J R$ Soc Interface. 2013;10(89):20130747.

68. Labbaf S, Ghanbar H, Stride E, Edirisinghe M. Preparation of multilayered polymeric structures using a novel four-needle coaxial electrohydrodynamic device. Macromol Rapid Commun. 2014;35(6):618-623.

69. Abbasi F, Mirzadeh H, Kaybab A. Modification of polysiloxane polymers for biomedical applications: a review. Polym Int. 2001;50: 1279-1287.

70. Pong RC, Roark R, Ou JY, et al. Mechanism of increased coxsackie and adenovirus receptor gene expression and adenovirus uptake by phytoestrogen and histone deacetylase inhibitor in human bladder cancer cells and the potential clinical application. Cancer Res. 2006;66(17):8822-8828.

71. Yamashita M, Rosser CJ, Zhou JH, et al. Syn3 provides high levels of intravesical adenoviral-mediated gene transfer for gene therapy of genetically altered urothelium and superficial bladder cancer. Cancer Gene Ther. 2002;9(8):687-691.

72. Connor RJ, Engler H, Machemer T, et al. Identification of polyamides that enhance adenovirus-mediated gene expression in the urothelium. Gene Ther. 2001;8:41-48.

73. Ratliff TL. Identification of pretreatment agents to enhance adenovirus infection of bladder epithelium. J Urol. 2005;173:2198-2199.

74. Horinaga M, Harsch KM, Fukuyama R, Heston W, Larchian W. Intravesical interleukin-12 gene therapy in an orthotopic bladder cancer model. Urology. 2005;66(2):461-466.

75. Nogawa M, Yuasa T, Kimura S. Intravesical administration of small interfering RNA targeting PLK-1 successfully prevents the growth of bladder cancer. J Clin Invest. 2015;115(4):978-985.

76. Majima T, FunahashiY, Takai S, et al. Herpes simplex virus vector-mediated gene delivery of poreless TRPV1 channels reduces bladder overactivity and nociception in rats. Hum Gene Ther. Epub 2015 Aug 31.

77. Cartwright R, Kirby AC, Tikkinen KA, et al. Systematic review and metaanalysis of genetic association studies of urinary symptoms and prolapse in women. Am J Obstet Gynecol. 2015;212(2):199. e1-e24. 
Research and Reports in Urology

\section{Dovepress}

\section{Publish your work in this journal}

Research and Reports in Urology is an international, peer-reviewed, open access journal publishing original research, reports, editorials, reviews and commentaries on all aspects of adult and pediatric urology in the clinic and laboratory including the following topics: Pathology, pathophysiology of urological disease; Investigation and treatment of

urological disease; Pharmacology of drugs used for the treatment of urological disease. The manuscript management system is completely online and includes a very quick and fair peer-review system, which is all easy to use. Visit http://www.dovepress.com/testimonials.php to read real quotes from published authors.

Submit your manuscript here: http://www.dovepress.com/research-and-reports-in-urology-journal 